\title{
Invariant Ellipsoids Method for Chaos Synchronization in a Class of Chaotic Systems
}

\author{
Giuseppe Fedele \\ Department of Informatics, Modeling, Electronics and System Engineering \\ University of Calabria, 87036, Rende, Italy \\ giuseppe.fedele@unical.it
}

\section{ARTICLE INFO}

\section{Article History}

Received December 14, 2021

Revised January 26, 2022

Accepted January 28, 2022

\section{Keywords}

Chaotic systems;

Ellipsoids;

Invariant sets;

LMI

\begin{abstract}
This paper presents an invariant sets approach for chaos synchronization in a class of master-slave chaotic systems affected by bounded perturbations. The method provides the optimal state-feedback gain in terms of the minimal ellipsoid that guarantees minimum synchronization error bound. The problem of finding the optimal invariant ellipsoid is formulated in terms of a semi-definite programming problem that can be easily solved using various simulation and calculus tools. The effectiveness of the proposed criterion is illustrated by numerical simulations on the synchronization of Chua's systems.
\end{abstract}

This is an open access article under the CC-BY-SA license.

\section{Introduction}

Chaotic dynamical systems exhibit sensitive dependence on the initial conditions and parameters value, i.e. small differences in initial conditions, such as those due to rounding errors in numerical computation, yield widely diverging outcomes for chaotic systems, making long-term prediction a difficult task.

Therefore, chaos synchronization between chaotic systems has attracted increasing interest in several fields of mathematics, physics and engineering systems due to its useful applications in chemical reactions [1], [2], [3], power converters [4], [5], biological systems [5], [6], [7], information processing [8,9], secure communication [10], [11], [12], just to name few.

Since the pioneering works of Pecora and Carroll [13], [14] it has become known that it is possible to force two chaotic systems to synchronize using a drive-response configuration. In fact, the chaos synchronization problem can be formulated in terms of two systems which oscillate in a synchronized manner. Given a chaotic system, which is considered as the master system, and another identical system, which is considered as the slave system, the chaos synchronization problem is to force the dynamical behaviors of these two systems to be identical after a transient time.

Over the last decades many different techniques for chaos control and synchronization have been developed. Adaptive control techniques are often used since in many applications the parameters of the controlled plant are unknown and/or the model of the system is not complete. In [15], for example, a simple, smooth and adaptive controller for resolving the control and synchronization problems of the modified Chua's circuit systems in case of unknown parameters is discussed.

A quadratic optimal regulator is used in [16] for synchronizing two complex chaotic systems in series form. Optimal control techniques allow to achieve least error with less control energy. In this 
case, the optimization on both energy and error can be easily realized. The synchronization of an uncertain slave system following the dynamic of an equivalent master system is developed in [17] by using a Lyapunov-based approach.

Linear state feedback by linear matrix inequalities (LMI) techniques have been proposed in [18], [19] where the synchronization criterion is transformed into LMI form which can be efficiently verified and solved. Most of the schemes to achieve chaos synchronization were originally designed for identical chaotic systems in which it is assumed that systems parameters are completely known in advance. Do to this unnatural assumption several approaches have been recently proposed to deal with this issue [20], [21], [22].

This paper proposes a static state-feedback control for master-slave synchronization designed by invariant ellipsoids techniques. The idea initially established in [23, 24, 25] and then further investigated over the last decades (see [26], [27] and the references therein) is based on the analysis of reachable and feasible sets for uncertain dynamic models or on the search for their approximations by simple convex domains like boxes, polyhedra or ellipsoids [28].

A set in the state space is said to be positively invariant for a given dynamical system if every trajectory initiated in this set remains inside it at all future time instants. In the presence of disturbances, if the invariance is preserved, the term of robust invariance is used.

The basic idea of the ellipsoidal control is to attract the state trajectory, for any initial state vector outside the ellipsoid, to a small ellipsoid region including the origin. When the trajectory of the system reaches the ellipsoid, it will remain within it for every future time instant. In this framework, the aim is therefore to synthesize a feedback gain that guarantees the synchronisation error belonging to the minimum invariant ellipsoid.

The main advantage of invariant ellipsoids lies in its simple characterization as a solution of LMI in terms of all the varying parameters, which is very convenient to be used in practice [29]. In fact, the control problem is reduced to semidefinite program, i.e., to the optimization of a linear function under LMI constraints.

Although the concept of invariant ellipsoid combined with LMI technique is widely used for analysis and design of control systems, however it found fewer applications in synchronization problems. For this reason, this paper exploits the theoretical framework of invariant ellipsoid control to address the synchronization between two chaotic systems.

The paper is organized as follows. Preliminaries definitions and results together with problem definition are presented in Sec. 2. Sec. 3 contains the main results consisting in finding a minimal invariant ellipsoid and the corresponding state-feedback control. A numerical simulation is reported in Sec. 4 to highlight the characteristics of the method. Finally some concluding remarks are included in the last section.

\section{Preliminaries}

Consider the master chaotic system described by

$$
\dot{x}=A x+g(x)+D w,
$$

where $x \in \mathbb{R}^{n}$ is the state vector, $A \in \mathbb{R}^{n \times n}$ is a constant matrix, $w \in \mathbb{R}^{n}$ is the exogenous disturbance which doesn't depend on the state vector $x$ and is bounded at each time instant as

$$
\|w(t)\| \leq 1, \quad \forall t \geq 0 .
$$

The quantity $D w$ takes into account both uncertainties on linear and nonlinear part of the chaotic system. The continuous nonlinear function $g(x)$ satisfies Lipschitz condition, namely

$$
\|g(x)-g(\hat{x})\| \leq \rho\|x-\hat{x}\|,
$$


where $\rho$ is the Lipschitz constant. Similar assumptions have been largely adopted in the devoted control literature. In general, as remarked in [20], [30], [31], the last assumption is not restrictive since the trajectories of chaotic systems are always bounded.

The slave chaotic system has the same structure of the master one with different initial conditions and with no information about the disturbance $w(t)$ :

$$
\dot{\hat{x}}=A \hat{x}+g(\hat{x})+u
$$

where the control input $u(t) \in \mathbb{R}^{n}$ is required to synchronize $\hat{x}(t)$ with the state of the master system $x(t)$. Based on the linear-state-feedback approach, the control input $u(t)$ is chosen as

$$
u=K(x-\hat{x})
$$

with $K \in \mathbb{R}^{n \times n}$. By defining the error $e(t)=x(t)-\hat{x}(t)$ which characterizes the synchronization accuracy, then

$$
\dot{e}=(A-K) e+D w+g(x)-g(\hat{x}) .
$$

The goal is to choose a suitable $K$, such that chaos synchronization is achieved, i.e. the error signal $e$ becomes as small as possible. With this aim we use the framework of invariant ellipsoids including the large deviation case as described in [32].

Remark 1 Note that the term Dw can be regarded both as uncertainty on the master and/or slave system. It is then the difference between the actual state of the system and the state modelled from the nominal model (4). Therefore what the method tries to do is to bring the synchronization closer to the actual state.

Definition 1 The ellipsoid

$$
\mathcal{E}=\left\{e \in \mathbb{R}^{n}: e^{T} P^{-1} e \leq\right\}, \quad P>0,
$$

is invariant for system (6), if two conditions hold:

1. (Small deviation case). $e(0) \in \mathcal{E}$ implies $e(t) \in \mathcal{E}$ for all $t \geq 0$.

2. (Large deviation case). $e(0) \notin \mathcal{E}$ implies $e(t) \rightarrow \mathcal{E}$ for $t \rightarrow \infty$ (in particular it can be $e(t) \in \mathcal{E}$ for $t \geq T$ with some $T>0$ ).

An ellipsoid $\mathcal{E}$ is minimal if it has the minimal sum of squares of its halfaxes, i.e. if $\operatorname{tr}(P)$ is minimal.

\subsection{Linear matrix inequalities}

A linear matrix inequality or LMI is a matrix inequality of the form

$$
F(x)=F_{0}+\sum x_{i} F_{i}>0,
$$

where $x_{1}, x_{2}, \ldots, x_{n}$ are the variables, $F_{i}=F_{i}^{T} \in \mathbb{R}^{n \times n}$ are given, and $F(x)>0$ means that $F(x)$ is positive-definite.

Lemma 1 Schur complements. For a given symmetric matrix

$$
M=\left[\begin{array}{cc}
Q(x) & S(x) \\
S(x)^{T} & R(x)
\end{array}\right]
$$


where $Q(x)=Q(x)^{T}, R(x)=R(x)^{T}$ and $S(x)$ depends affinely on $x$, Schur complements states that the $L M I M>0$ is equivalent to the matrix inequalities

$$
R(x)>0, \quad Q(x)-S(x) R(x)^{-1} S(x)^{T}>0
$$

or, equivalently,

$$
Q(x)>0, \quad R(x)-S(x)^{T} Q(x)^{-1} S(x)>0 .
$$

Lemma $2 S$-Lemma. Let $A, B$ be symmetric $n \times n$ matrices, and assume that the quadratic inequality $x^{T} A x \geq 0$ is strictly feasible (there exists $\bar{x}$ such that $\bar{x}^{T} A \bar{x}>0$ ). Then the quadratic inequality $x^{T} B x \geq 0$ is a consequence of it, i.e.

$$
x^{T} A x \geq 0 \rightarrow x^{T} B x \geq 0
$$

if and only if there exists a non-negative $\tau$ such that

$$
B \geq \tau A \text {. }
$$

Lemma 3 Matrix square root. Let $F$ be a positive definite Hermitian matrix. Then $F$ has a diagonalization

$$
F=P^{*} \operatorname{diag}\left\{\lambda_{1}, \ldots, \lambda_{n}\right\} P
$$

where $P$ is a unitary matrix and $\lambda_{1}, \ldots, \lambda_{n}$ are the eigenvalues of $F$, which are all positive. The square root of $F$ is defined as the matrix

$$
F^{1 / 2}=P^{*} \operatorname{diag}\left\{\sqrt{\lambda_{1}}, \ldots, \sqrt{\lambda_{n}}\right\} P .
$$

\section{Invariant ellipsoid design}

In order to reduce the synchronization error, the dynamic of which is defined in (6), the optimal state-feedback gain $K$ is computed in this section. This controller gain $K$, obtained in the following theorem, gives the minimum invariant ellipsoid, expressed as the minimum of the trace of $P$, for the error between master and slave trajectories. The error is reduced both in the case when initial error started within the smallest invariant ellipsoid and in the large deviation case, i.e. when the initial error is outside the optimal invariant set.

Theorem 1 Let $M=M^{T} \in \mathbb{R}^{n \times n}$ be the matrix such that

$$
M M^{T}=\tau I+D D^{T}
$$

where $\tau \geq 0$, i.e. $M$ is the square root of the positive definite matrix on the r.h.s. The solution $\hat{Q}$ and $\hat{Y}$ of the minimization problem

$$
\min \operatorname{tr}(H)
$$

subject to

$$
\begin{gathered}
\left(\begin{array}{cc}
A^{T} Q+Q A-Y-Y^{T}+\rho^{2} I+\tau Q & Q M \\
M^{T} Q & -\tau I
\end{array}\right) \leq 0, \\
\left(\begin{array}{cc}
H & I \\
I & Q
\end{array}\right) \geq 0,
\end{gathered}
$$

on matrix variables $Q=Q^{T}, H=H^{T}, Y \in \mathbb{R}^{n \times n}$ defines the matrix $\hat{P}=\hat{Q}^{-1}$ of the minimal invariant ellipsoid and corresponding filter gain

$$
\hat{K}=\hat{Q}^{-1} \hat{Y} .
$$


Proof. To satisfy both properties of invariant ellipsoid (7) it is sufficient to suppose that there exists a quadratic Lyapunov function

$$
V(e)=e^{T} Q e, Q=Q^{T} \in \mathbb{R}^{n \times n}, Q>0
$$

with $Q=P^{-1}$, such that

$$
\dot{V}(e) \leq 0, \forall(e, w): V(e) \geq 1, w^{T} w \leq 1
$$

Differentiating $V$ along the error dynamical trajectory (6) and using (3) yields

$$
\begin{aligned}
\dot{V}(e) & =e^{T}\left[A_{K}^{T} Q+Q A_{K}\right] e+2 e^{T} Q D w+2 e^{T} Q(g(x)-g(\hat{x})) \leq \\
& \leq e^{T}\left[A_{K}^{T} Q+Q A_{K}\right] e+2 e^{T} Q D w+2 \rho\|Q e\|\|e\|
\end{aligned}
$$

where $A_{K}=A-K$.

Since $2 \rho\|Q e\|\||| \mid \leq\| Q e\left\|^{2}+\rho^{2}\right\| e \|^{2}$ then it follows that

$$
\begin{array}{r}
e^{T}\left[A_{K}^{T} Q+Q A_{K}+Q Q+\rho^{2} I\right] e+2 e^{T} Q D w \leq 0, \\
\forall(e, w): e^{T} Q e \geq 1, w^{T} w \leq 1 .
\end{array}
$$

Due to Schur complements the first inequality in (16) can be rewritten as

$$
\left(\begin{array}{c}
e \\
w
\end{array}\right)^{T}\left(\begin{array}{cc}
A_{K}^{T} Q+Q A_{K}+Q Q+\rho^{2} I & Q D \\
D^{T} Q & 0
\end{array}\right)\left(\begin{array}{c}
e \\
w
\end{array}\right) \leq 0 .
$$

Also $e^{T} Q e \geq 1$ and $w^{T} w \leq 1$ can be formulated as

$$
\left(\begin{array}{c}
e \\
w
\end{array}\right)^{T}\left(\begin{array}{cc}
Q & 0 \\
0 & I
\end{array}\right)\left(\begin{array}{c}
e \\
w
\end{array}\right) \geq 0 .
$$

From S-Lemma it follows that (18) implies (17) if exists $\tau \geq 0$ such that

$$
-\left(\begin{array}{cc}
A_{K}^{T} Q+Q A_{K}+Q Q+\rho^{2} I & Q D \\
D^{T} Q & 0
\end{array}\right) \geq \tau\left(\begin{array}{cc}
Q & 0 \\
0 & I
\end{array}\right) .
$$

Hence

$$
\left(\begin{array}{cc}
A_{K}^{T} Q+Q A_{K}+Q Q+\rho^{2} I+\tau Q & Q D \\
D^{T} Q & -\tau I
\end{array}\right) \leq 0 .
$$

By Schur complements Eq. (20) is equivalent to

$$
A_{K}^{T} Q+Q A_{K}+\rho^{2} I+\tau Q+\frac{1}{\tau} Q\left(\tau I+D D^{T}\right) Q \leq 0
$$

from which (10) follows when $Y=Q K$.

To reduce minimization of $\operatorname{tr}\left(Q^{-1}\right)$ to a linear problem introduce a matrix $H=H^{T}$ such that $Q^{-1} \leq H$. Due to Schur complements this inequality is equivalent to LMI (11). Therefore the problem is reduce to the minimization of $\operatorname{tr}(H)$ subject to (10) and (11) and the proof follows.

Remark 2 The introduction of the new variable $\tau$, due to the S-procedure, makes (10) a bilinear matrix inequality (BMI). Because $\tau$ is a scalar, its optimal value can be found by executing a simple loop or by using a BMI solver. For $\tau$ fixed, the problem defined by (9)-(11) is a semidefinite programming problem (SDP). 


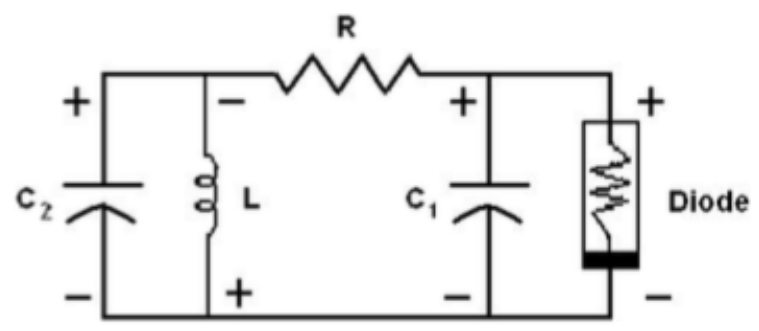

Fig. 1. The Chua system.

\section{Numerical example}

In this section the Chua circuit is simulated to illustrate the above control method for chaos synchronization. The simulation is performed within the Matlab 2021a environment while the underlying optimisations are carried out using the Yalmip toolbox [33]. Chua's circuit in Fig. 1 is the simplest and most widely studied real nonlinear dynamical systems [34]. It consists of three energy-store elements (an inductor and two capacitors), a linear resistor and a single nonlinear resistor, called Chua's diode. Using the Kirchhoff's circuit laws the circuit is described by equations:

$$
\begin{aligned}
C_{1} \frac{d v_{C 1}}{d t} & =\frac{1}{R}\left(v_{C 2}-v_{C 1}\right)-\phi\left(v_{C 1}\right), \\
C_{2} \frac{d v_{C 2}}{d t} & =\frac{1}{R}\left(v_{C 1}-v_{C 2}\right)+i_{L}, \\
L \frac{d i_{L}}{d t} & =-v_{C 2}
\end{aligned}
$$

where $R$ is a linear resistance, $v_{C 1}$ and $v_{C 2}$ are the voltages across capacitors $C_{1}$ and $C_{2}$, respectively, $i_{L}$ is the current through the inductor $L$ and $\phi\left(v_{C 1}\right)$ is the current through the nonlinear resistor as a function of the voltage across capacitor $C_{1}$. This nonlinear function is described by the oddsymmetric piecewise-linear function

$$
\phi\left(v_{C 1}\right)=G_{b} v_{C 1}+\frac{G_{a}-G_{b}}{2}\left(\left|v_{C 1}+B_{p}\right|-\left|v_{C 1}-B_{p}\right|\right),
$$

where $G_{a}, G_{b}$ and $B_{p}$ are three fixed constants of the diode. In particular $G_{a}$ is the slope of the nonlinear function in the inner region and $G_{b}$ the slope in the two outer regions.

The system (22) can be rewritten in dimensionless form as

$$
\begin{aligned}
\frac{d x_{1}}{d \tau} & =k \alpha\left(-x_{1}+x_{2}-f\left(x_{1}\right)\right), \\
\frac{d x_{2}}{d \tau} & =k\left(x_{1}-x_{2}+x_{3}\right), \\
\frac{d x_{3}}{d \tau} & =-k \lambda x_{2},
\end{aligned}
$$

with

$$
f(x)=\bar{b} x+\frac{\bar{a}-\bar{b}}{2}(|x+1|-|x-1|)
$$

where

$$
\begin{array}{ccc}
x_{1}=\frac{v_{C 1}}{B_{p}}, & x_{2}=\frac{v_{C 2}}{B_{p}}, & x_{3}=\frac{R i_{L}}{B_{p}}, \\
\alpha=\frac{C_{1}}{C_{1}}, & \lambda=\frac{C_{2} R^{2}}{L}, & \\
\bar{a}=R G_{a}, & \bar{b}=R G_{b}, & \tau=\frac{t}{\left|R C_{2}\right|}
\end{array}
$$




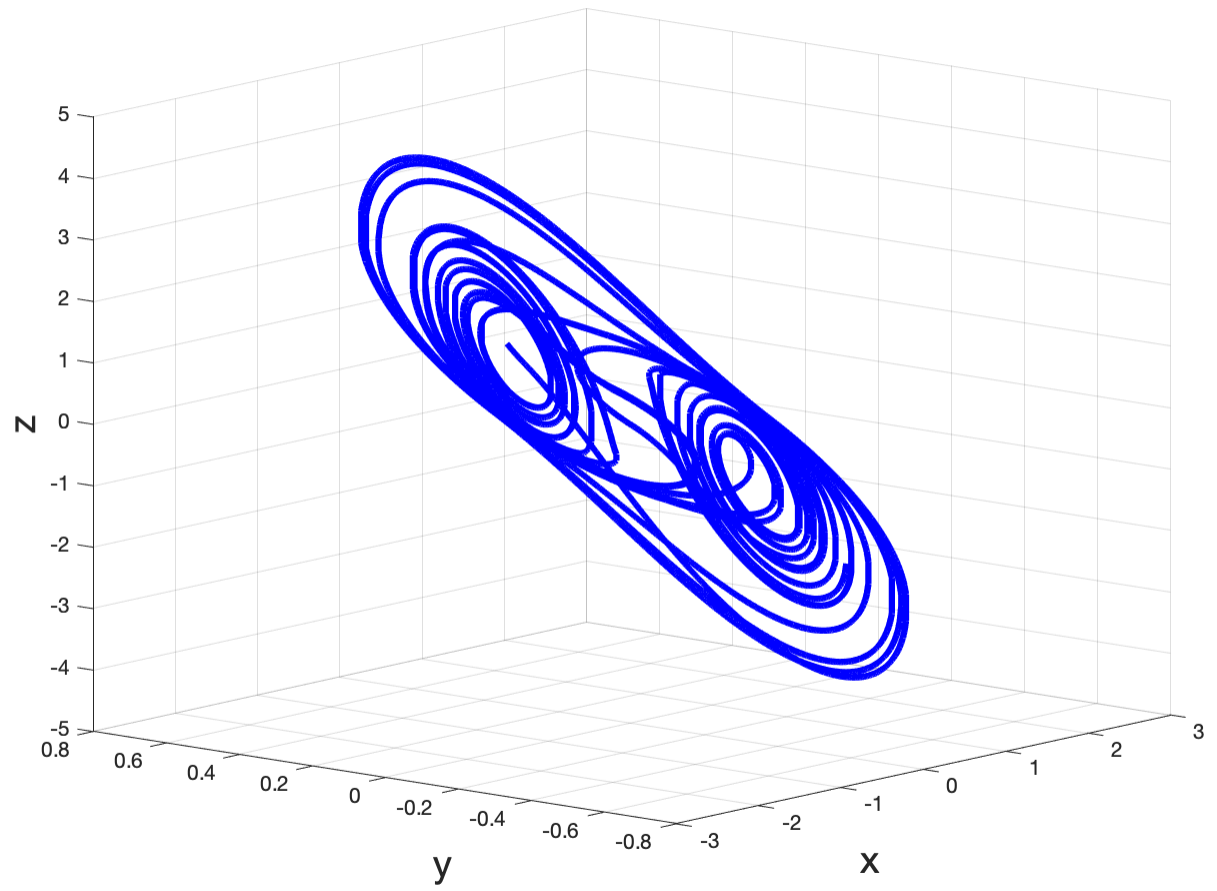

Fig. 2. Chua's system $\left(\alpha=15.6, \lambda=28, m_{0}=-1.143, m_{1}=-0.714\right)$ : the case of double-scroll attractor.

and

$$
\begin{array}{ll}
k=1 & \text { if } \quad R C_{2}>0, \\
k=-1 & \text { if } \quad R C_{2}<0 .
\end{array}
$$

Throughout the rest of the section, we will use the dimensionless form with $k=1$ rewritten in matrix form as

$$
\dot{x}=A x+\phi(x),
$$

where

$$
A=\left(\begin{array}{ccc}
-\alpha & \alpha & 0 \\
1 & -1 & 1 \\
0 & -\lambda & 0
\end{array}\right), \quad \phi(x)=\left(\begin{array}{c}
-\alpha\left(m_{1} x_{1}+\frac{m_{0}-m_{1}}{2}\left(\left|x_{1}+1\right|-\left|x_{1}-1\right|\right)\right) \\
0 \\
0
\end{array}\right) .
$$

If $\alpha=15.6, \lambda=28, m_{0}=-1.143$ and $m_{1}=-0.714$ then the Chua system exhibits a double scroll attractor (Fig. 2).

The Lipschitz constant $\rho=20$ satisfies (3). The uncertainty on the master system is expressed in terms of the product $D w$ with $D=\operatorname{diag}[1,2,3]$ and the unit norm vector $w$ with each component uniformly distributed in $[-1,1]$. The initial condition of the slave is assumed to be $\hat{x}=(3,-2,5)^{T}$. The optimal value of $\tau=93.697$ in the interval $[0.1,200]$ is found by simple loop of the main SDP problem solver with a fixed value of $\tau$. For this $\tau$ the minimum ellipsoid is characterized by the matrix $P$

$$
P=\left(\begin{array}{ccc}
2.97 \times 10^{-5} & 4.38 \times 10^{-9} & -1.16 \times 10^{-12} \\
4.38 \times 10^{-9} & 3.01 \times 10^{-5} & -6.99 \times 10^{-9} \\
-1.16 \times 10^{-12} & -6.99 \times 10^{-9} & 3.06 \times 10^{-5}
\end{array}\right)
$$


having trace equal to $9.04 \times 10^{-5}$. In this case the optimal control law has the following feedback gain matrix

$$
K=\left(\begin{array}{ccc}
1.70 \times 10^{4} & 5.82 & 0 \\
5.84 & 1.73 \times 10^{4} & -9.30 \\
0 & -9.36 & 1.78 \times 10^{4}
\end{array}\right)
$$

To Trajectories of master and slave systems are depicted in Fig. 3.
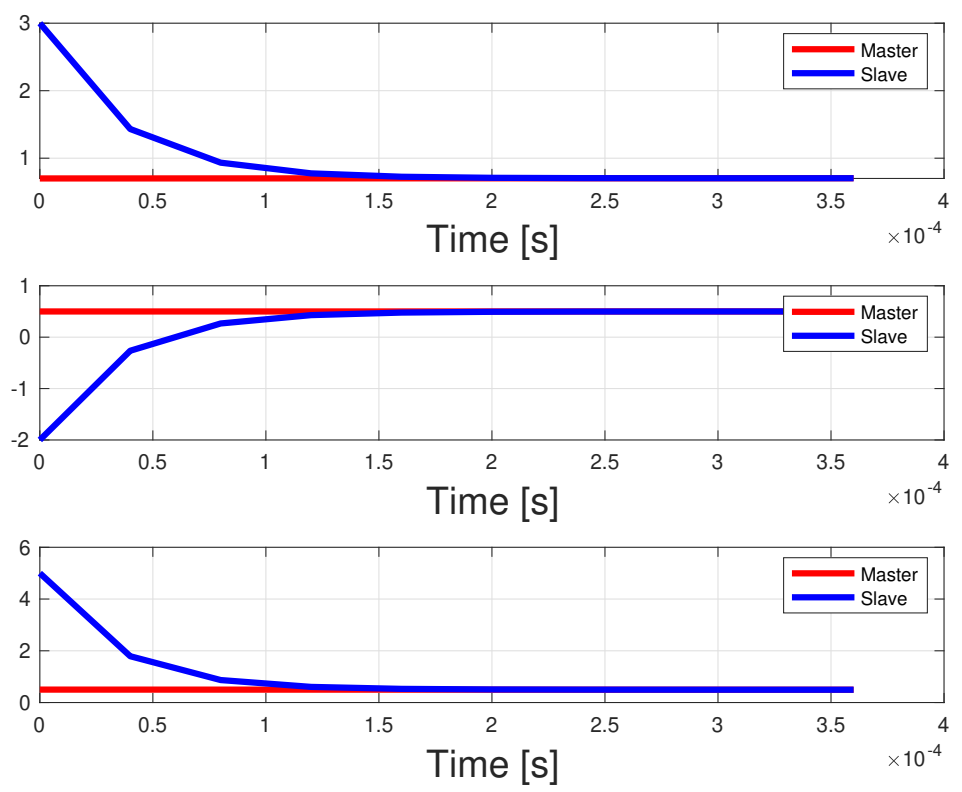

Fig. 3. Master and slave outputs.

\section{Conclusions}

A robust state-feedback controller has been proposed for chaos synchronization in a class of chaotic systems. It makes use of the method of invariant ellipsoids which yields the optimal solution in terms of minimal ellipsoid containing the synchronization error. Robustness refers to the ability to maintain the invariance even in the presence of disturbances on the master system. The effectiveness of the proposed approach has been tested in the case of Chua master-slave systems synchronization. Two interesting perspectives are the extension of this method to the case of systems with interval uncertainties and its application to the synchronisation of systems of different types and dimensions.

Funding: This research received no external funding.

Conflicts of Interest: The authors declare no conflict of interest.

\section{References}

[1] I. R. Epstein and K. Showalter, "Nonlinear Chemical Dynamics: Oscillations, Patterns, and Chaos," J. Phys. Chem., vol. 100, pp. 13132-13147, 1996, https://doi.org/10.1021/jp953547m.

[2] H. Fatoorehchi, R. Zarghami, H. Abolghasemi, and R. Rach, "Chaos control in the cerium-catalyzed Belousov-Zhabotinsky reaction using recurrence quantification analysis measures," Chaos, Solitons and Fractals, vol. 76, pp. 121-129, 2015, https://doi.org/10.1016/j.chaos.2015.03.019. 
[3] M. Lis, S. Onuma, D. Przyczyna, P. Zawal, T. Mazur, K. Pilarczyk, and K. Szaciłowski, "From Oscillatory Reactions to Robotics: A Serendipitous Journey Through Chemistry, Physics and Computation," Handbook of Unconventional Computing, pp. 1-79, 2021, https://doi.org/10.1142/9789811235740_0001.

[4] L. Wang, Z. Meng, Y. Sun, and L. Guo, "Bifurcation and chaos analysis of power converter for switched reluctance motor drive," Int. Journal of Electronics, vol. 104, 1, pp. 157-173, 2017, https://doi.org/10. 1080/00207217.2016.1196745.

[5] H. Zhang, S. Dong, and W. Guan, "Analysis of Fast-Scale Bifurcation in Peak Current Controlled BuckBoost Inverter Based on Unified Averaged Model,” Int. J. Bifurcation Chaos, vol. 26, no. 5, p. 1650074, 2016, https://doi.org/10.1142/S0218127416500747.

[6] F. Borgonovi, F. M. Izrailev, L. F. Santos, and V. G. Zelevinsky, "Quantum chaos and thermalization in isolated systems of interacting particles," Physics Reports, vol. 626, pp. 1-58, 2016, https://doi.org/10. 1016/j.physrep.2016.02.005.

[7] J. Hettich and J. C. M. Gebhardt, "Periodic synchronization of isolated network elements facilitates simulating and inferring gene regulatory networks including stochastic molecular kinetics," BMC bioinformatics, vol. 23, no. 1, pp. 1-30, 2022, https://doi.org/10.1186/s12859-021-04541-6.

[8] R. Lamb, A. Cavagnetto, and T. Akmal, "Examination of the Nonlinear Dynamic Systems Associated with Science Student Cognition While Engaging in Science Information Processing," Int. J. of Sci. and Math. Educ., vol. 14, no.1, pp. 187-205, 2016, https://doi.org/10.1007/s10763-014-9593-2.

[9] R. Guesmi, M. A. B. Farah, A. Kachouri, and M. Samet, "A novel chaos-based image encryption using DNA sequence operation and Secure Hash Algorithm SHA-2," Nonlinear Dynamics, vol. 83, no. 3, pp. 1123-1136, 2016, https://doi.org/10.1007/s11071-015-2392-7.

[10] B. Wang, S. M. Zhong, and X. C. Dong, "On the novel chaotic secure communication scheme design," Commun. Nonlinear Sci. Numer. Simulat., vol. 39, pp. 108-117, 2016, https://doi.org/10.1016/j.cnsns. 2016.02.035.

[11] L. Runzi and W. Yinglan, "Finite-time stochastic combination synchronization of three different chaotic systems and its application in secure communication," Chaos: An Interdisciplinary Journal of Nonlinear Science, vol. 22, pp. 023109, 2012, https://doi.org/10.1063/1.3702864.

[12] F. Aliabadi and M.H. Majidi, S. Khorashadizadeh, "Chaos synchronization using adaptive quantum neural networks and its application in secure communication and cryptography," Neural Computing and Applications, pp. 1-13, 2022, https://doi.org/10.1007/s00521-021-06768-z.

[13] L. M. Pecora and T. L. Carroll, "Synchronization in chaotic systems," Phys. Rev. Lett., vol. 64, pp. 821824, 1990, https://doi.org/10.1103/PhysRevLett.64.821.

[14] L. M. Pecora, T. L. Carroll, G. A. Johnson, D. J. Mar, and J. F. Heagy, "Fundamentals of synchronization in chaotic systems, concepts, and applications," Chaos: An Interdisciplinary Journal of Nonlinear Science, vol. 7, pp. 520-543, 1997, https://doi.org/10.1063/1.166278.

[15] M. T Yassen, "Adaptive control and synchronization of a modified Chua's circuit system," Appl. Math. and Comp., vol. 135, pp. 113-128, 2003, https://doi.org/10.1016/S0096-3003(01)00318-6.

[16] Z. M. Ge and C. H. Yang, "Chaos synchronization and chaotization of complex chaotic systems in series form by optimal control," Chaos, Solitons and Fractals, vol. 42, pp. 994-1002, 2009, https://doi.org/10. 1016/j.chaos.2009.02.026.

[17] G. Fedele, A. Ferrise, and F. Chiaravalloti, "Uncertain master-slave synchronization with implicit minimum saturation level," Appl. Math. Modelling, vol. 40, pp. 1193-1198, 2016, https://doi.org/10.1016/j. apm.2015.07.010.

[18] G. P. Jiang and W. X. Zheng, "An LMI criterion for linear-state feedback based chaos synchronization of a class of chaotic systems," Chaos, Solitons and Fractals, vol. 26, pp. 437-443, 2005, https://doi.org/10. 1016/j.chaos.2005.01.012.

[19] F. Chen and W. Zhang, "LMI criteria for robust chaos synchronization of a class of chaotic systems," Nonlinear Analysis, vol. 67, pp. 3384-3393, 2007, https://doi.org/10.1016/j.na.2006.10.020.

[20] M. P. Aghababa and H. P. Aghababa, "A general nonlinear adaptive control scheme for finite-time synchronization of chaotic systems with uncertain parameters and nonlinear inputs," Nonlinear Dynamics, vol. 69, pp. 1903-1914, 2012, https://doi.org/10.1007/s11071-012-0395-1. 
[21] B. B. Sharma and I. N. Kar, "Contraction theory based adaptive synchronization of chaotic systems," Chaos, Soliton \& Fractals, vol. 41, pp. 2437-2447, 2009, https://doi.org/10.1016/j.chaos.2008.09.031.

[22] M. Pourmahmood, S. Khanmohammadi, and G. Alizadeh, "Synchronization of two different uncertain chaotic systems with unknown parameters using a robust adaptive sliding mode controller," Commun. Nonlinear Sci. Numer. Simulat., vol. 16, pp. 2853-2868, 2011, https://doi.org/10.1016/j.cnsns.2010.09. 038.

[23] D. P. Bertsekas and I. B. Rhodes, "On the minimax reachability of target sets and target tubes," Automatica, vol. 7, pp. 233-247, 1971, https://doi.org/10.1016/0005-1098(71)90066-5.

[24] D. P. Bertsekas and I. B. Rhodes, "Recursive state estimation for a set-membership description of uncertainty," IEEE Trans. on Aut. Contr., vol. 16, pp. 117-128, 1971, https://doi.org/10.1109/TAC.1971. 1099674.

[25] F. C. Schweppe, Uncertain Dynamic Systems, Prentice Hall, Englewood Cliffs (N. J.), 1973, https://books. google.co.id/books/about/Uncertain_Dynamic_Systems.html?id=BfdQAAAAMAAJ.

[26] F. Blanchini and S. Miani, Set-Theoretic Methods in Control, Birkhauser - Boston, Basel-Berlin, 2007, https://doi.org/10.1007/978-0-8176-4606-6.

[27] H. Yang, Y. Zhang, W. Gu, F. Yang, and Z. Liu, "Set-membership filtering for automatic guided vehicles with unknown-but-bounded noises," Transactions of the Institute of Measurement and Control, vol. 44, no. 3, pp. 716-725, 2022, https://doi.org/10.1177/01423312211043666.

[28] B. T. Polyak, S. A. Nazin, C. Durieu, and E. Walter, "Ellipsoidal parameter or state estimation under model uncertainty," Automatica, vol. 40, pp. 1171-1179, 2004, https://doi.org/10.1016/j.automatica.2004. 02.014.

[29] S. Boyd, L. El Ghaoui, E. Ferron, and V. Balakrishnan, Linear Matrix Inequalities in System and Control Theory, Philadelphia: SIAM, 1994, https://doi.org/10.1137/1.9781611970777.

[30] H. Kebriaei and M. J. Yazdanpanah, "Robust adaptive synchronization of different uncertain chaotic systems subject to input nonlinearity," Commun. Nonlinear Sci. Numer. Simulat., 15, pp. 430-441, 2010 , https://doi.org/10.1016/j.cnsns.2009.04.005.

[31] W. Yu, G. Chen, and J. Lü, “On pinning synchronization of complex dynamical networks,” Automatica, vol. 45, pp. 429-435, 2009, https://doi.org/10.1016/j.automatica.2008.07.016.

[32] B. T. Polyak and M. V. Topunov, "Filtering with nonrandom noise: invariant ellipsoids technique," 17th World Congress IFAC, 2008, https://doi.org/10.3182/20080706-5-KR-1001.02596.

[33] A. K. Ravat, A. Dhawan, and M. Tiwari, "LMI and YALMIP: Modeling and Optimization Toolbox in MATLAB," Advances in VLSI, Communication, and Signal Processing, pp. 507-515, 2021, https://doi. org/10.1007/978-981-15-6840-4_41.

[34] T. Matsumoto, L. O. Chua, and M. Komuro, "The Double Scroll," IEEE Trans. Circuits Syst., vol. 32, no. 8, pp. 798-818, 1985, https://doi.org/10.1109/TCS.1985.1085791. 